American J. of Engineering and Applied Sciences 3 (4): 678-682, 2010

ISSN 1941-7020

(C) 2010 Science Publications

\title{
The Solar Tracking System by Using Digital Solar Position Sensor
}

\author{
Singthong Pattanasethanon \\ Faculty of Engineering, Mahasarakham University \\ Kamreang, Kantharawichai, Mahasarakham, 44150 Thailand
}

\begin{abstract}
Problem statement: An optimal control on two axes and design for solar tracker which called altitude and azimuth is challenge. Approach: The phototransistor with the shade that blocks the screen was employed as a detector of solar beam radiation. The height of the screen determined the sensitivity operation or period of tracking in this solar tracker. The phototransistor is particularly designed to detect solar bean radiation thoroughly through the two axes with the operating time. The mechanism of this solar tracker is that it has a capacity of solar tracking in every $10 \mathrm{~min}$, approximately, which respond in terms of time at about $37^{\circ} \mathrm{sec}^{-1}$ with and operating point at $0.3 \mathrm{sec}$. Results: Our solar tracker obtained an average deviation at about $2.5^{\circ} \mathrm{h}^{-1}$. In weak sunlight however, the value varies and fluctuates rapidly depending on sky condition. Conclusion: The accuracy of solar position tracking function satisfied our goal as well. There is only average of $2.5^{\circ}$ error shown. The experiment also shows that the error rate diminishes as the solar radiance expand. However, we hope to develop this device to be more exact in the position.
\end{abstract}

Key words: Solar tracker, altitude and azimuth angle, beam radiation, optimal control

\section{INTRODUCTION}

From the past decades, several techniques and experiment conduction has been developed for solar tracking device for an alternative source of energy, solar energy (ElGizawy et al., 2010; Marghany and Hashim, 2009). Based on the tracking principles (Roth et al., 2004), we can classify the controlling process into 3 processes namely passive control unit, microprocessor control unit and electro-optical control unit (Konar and Mandal, 1991). Passive control unit is basically a system conducted without any electronic device. It engages in the stage of change in liquid substance. The liquid masses lift the solar cell in motion in order to track the solar energy. The advantage of this controlling unit is that it does not require any electricity to receive solar energy and it is less fragile. However, the accuracy is quite low. On the other hand, microprocessor control unit is the opposite. It is more accurate. In addition, it tracks solar energy by microprocessors and computes the solar position with the equation. Nonetheless, the device is more expensive and requires users' nurture. Finally, electro-optical control unit tracks the sun by a solar detecting device that is sensitive to solar radiance. Simple equipment such as photo diodes, phototransistors and LDR are employed in the device of our working station (Koyuncu and Balasubramanian, 1991; Kalogirou, 1996). The system might not study under the cloudy sky condition or the lack-of-sunlight condition because less solar energy diminishes the efficiency of the device. On the contrary, it is not necessary to detect solar radiance all the time. We only start the tracking function when the appropriate time approaches. For instance, the period of time when it is plausible to obtain the highest solar energy during the day.

Devices setup and design: The device uses the shading method to detect solar energy with a direct circuit motor controlling the whole system created with photodiodes. The photodiode faced perpendicularly to the sun, permanently. The design is sub divided into two categories; detecting circuit, driver motor circuit.

Solar tracking devices: In this study, we select phototransistor as the solar detecting device. The semantic of solar detection is the solar position in this case. The altitude direction of the detection addresses the solar position by the shade of parabolic shade. As shown in Fig. 1a, when the semantic of solar position is parallel to the phototransistors, the parabolic shape phototransistors will receive solar energy in the ' $\mathrm{ON}$ ' condition. In addition, when the sun orbits to its former position, the shades will form a shadow from and the phototransistor status will be on the 'OFF' condition. Note that the 'OFF' condition denotes little to no current flow in the system. Consider the semantic ' $\mathrm{ON}$ ' status as ' 1 ' and 'OFF' status as ' 0 '. The phototransistor facing direction is $60^{\circ}$. 


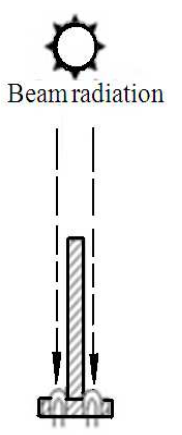

Phototransistor

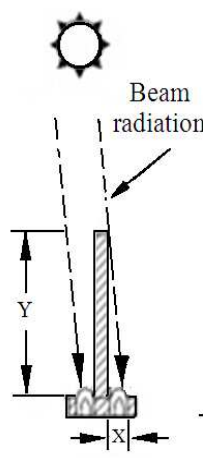

Phototransistor

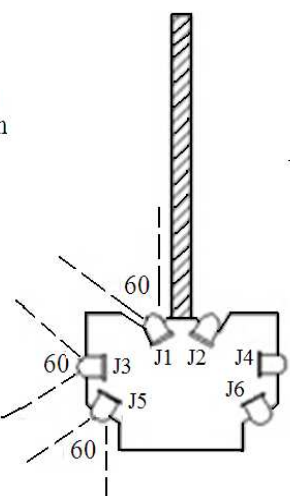

(b)
Fig. 1: Sensor; (a) light balance and unbalance; (b) Photo Tr. Configuration

Therefore, in order to detect solar energy all the way through the altitude, we designed our device to contain 6 phototransistors; $\mathrm{J}_{1}, \mathrm{~J}_{2}, \mathrm{~J}_{3}, \mathrm{~J}_{4}, \mathrm{~J}_{5}$ and $\mathrm{J}_{6}$. As shown in Fig. 1b, the characteristic of this design assures that the device is capable of detecting solar energy whenever it begins. The height of the shade ' $y$ ' is computed as the equation below.

$\mathrm{Y}=\frac{\mathrm{X}}{\tan \theta}$

Where:

$\mathrm{x}=$ The distance from the shade to the edge of the phototransistor

$\theta=$ The degrees angle of the sun which moves from its original position

The digital circuit constructs Table 1 with an arithmetic Boolean logic as expressed in Eq. 2 and 3. From both equations, we obtain a logic gate equation as displayed in Fig. 2. Where, $\mathrm{Q}_{1}$ and $\mathrm{Q}_{2}$ is the output of the solar detecting circuit which begins the motor in the altitude direction:

$$
\mathrm{Q}_{1}=\mathrm{J}_{1} \overline{\mathrm{J}}_{2}+\overline{\mathrm{J}}_{2} \overline{\mathrm{J}}_{3} \mathrm{~J}_{4}
$$

$$
\mathrm{Q}_{2}=\mathrm{J}_{2} \overline{\mathrm{J}}_{1}+\overline{\mathrm{J}}_{1} \overline{\mathrm{J}}_{4} \mathrm{~J}_{1}
$$

The light detector circuit in Fig. 2 is able to implement as the print circuit board which illustrated in Fig. 3.

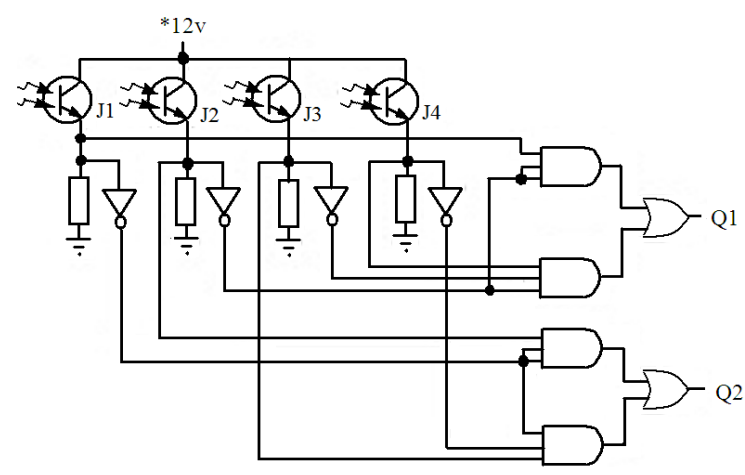

Fig. 2: Light detector circuit in solar altitude direction

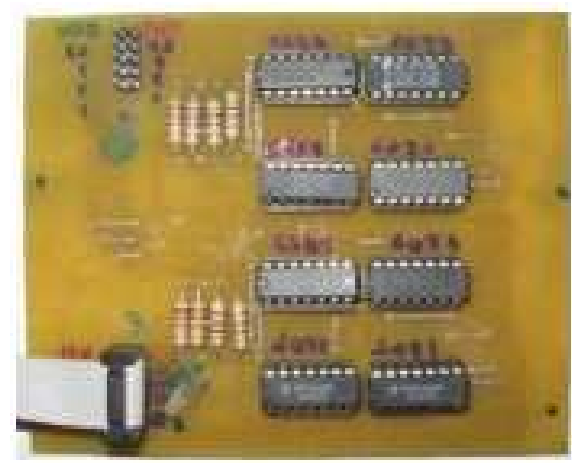

Fig. 3: Print circuit board of the light detector circuit

\begin{tabular}{|c|c|c|c|c|c|}
\hline \multicolumn{4}{|c|}{ Input } & \multicolumn{2}{|c|}{ Output } \\
\hline $\mathrm{J}_{1}$ & $\mathbf{J}_{2}$ & $\mathrm{~J}_{3}$ & $\mathrm{~J}_{4}$ & $\mathrm{Q}_{1}$ & $\mathrm{Q}_{2}$ \\
\hline 0 & 0 & 0 & 0 & 0 & 0 \\
\hline 0 & 0 & 0 & 1 & 1 & 0 \\
\hline 0 & 0 & 1 & 0 & 0 & 1 \\
\hline 0 & 0 & 1 & 1 & 0 & 0 \\
\hline 0 & 1 & 0 & 0 & 0 & 1 \\
\hline 0 & 1 & 0 & 1 & 0 & 1 \\
\hline 0 & 1 & 1 & 0 & 0 & 1 \\
\hline 0 & 1 & 1 & 1 & 0 & 1 \\
\hline 1 & 0 & 0 & 0 & 1 & 0 \\
\hline 1 & 0 & 0 & 1 & 1 & 0 \\
\hline 1 & 0 & 1 & 0 & 1 & 0 \\
\hline 1 & 0 & 1 & 1 & 1 & 0 \\
\hline 1 & 1 & 0 & 0 & 0 & 0 \\
\hline 1 & 1 & 0 & 1 & 0 & 0 \\
\hline 1 & 1 & 1 & 0 & 0 & 0 \\
\hline 1 & 1 & 1 & 1 & 0 & 0 \\
\hline
\end{tabular}

Table 1: True table of light detection on solar altitude direction

The semantic of the azimuth direction is most likely compatible to altitude direction. It also developed six phototransistors namely $\mathrm{K} 1, \mathrm{~K} 2, \mathrm{~K} 3, \mathrm{~K} 4, \mathrm{~K} 5$ and K6. 

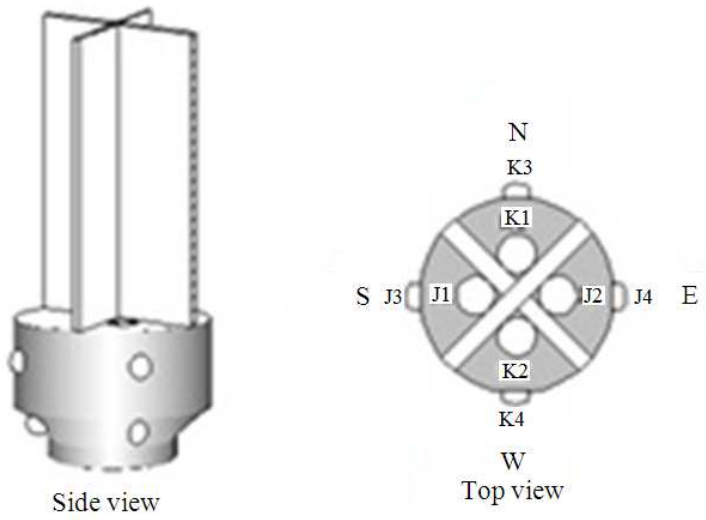

Fig. 4: Position of phototransistor configuration on base

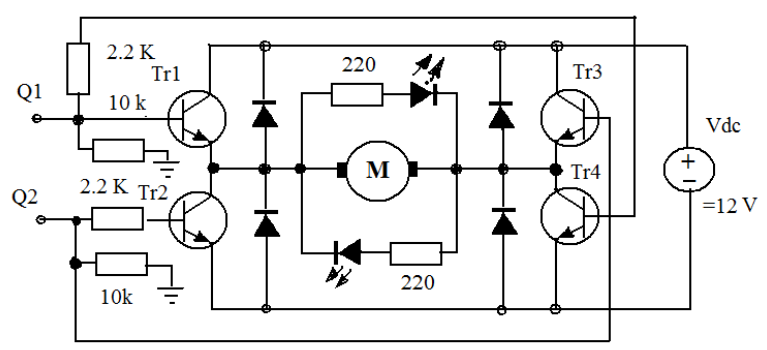

Fig. 5: Motor driver circuit for solar altitude direction

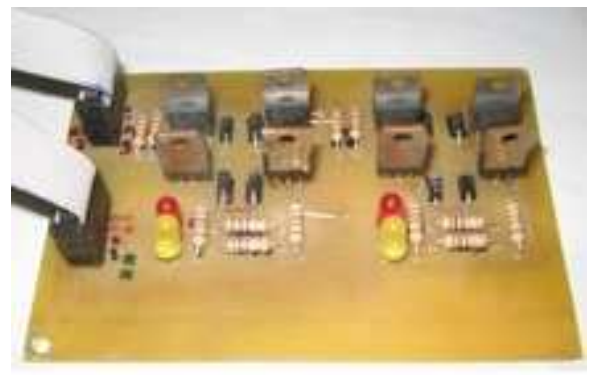

Fig. 6: Print circuit board of motor driver circuit

The structure and design is at the same position with the altitude as well, as shown in Fig. 4.

Motor driver circuit: This experiment is conducted by the main source of power or motor which functions both altitude and azimuth directions. Two 12 volt direct circuit motors are employed in the device. The motor test from head gear indicates that the motor speed is at $8 \mathrm{rpm}$. This part is split into two significant parts which are the altitude part and azimuth part. The motor structure is as displayed in the Fig. 5 and 6.

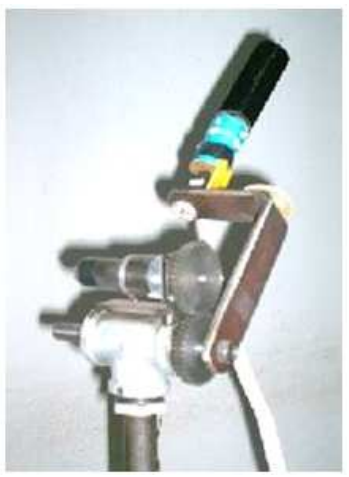

(a)

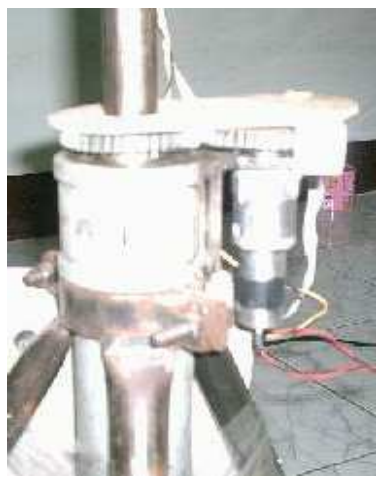

(b)
Fig. 7: Structure mechanism of solar tracking; (a) altitude movement direction; (b) azimuth movement direction

Structure: The structure is divided into half. The mechanism of the first half or altitude is that the sensor must be attached to a shade metal in a perpendicular direction. The motor obtains a ratio of gear at 1:1 in the axle of the motor as in Fig. 7a. The azimuth function consists of a metal pipe with a $5 \mathrm{~cm}$. diameter and $30 \mathrm{~cm}$. in length. Place two bullets both head and tail of the rotating stand. The same type of motor is being used in the axle.

Device testing: The testing semantic for this device consists of two significant tasks; the time feedback response and the accuracy of the device. A 100-watt bulb is part of this experiment test. We test this solar parabolic tracker device by tracking the 100 watts bulb and its position. This process is done in order to evaluate the speed of this device in terms of its tracking function.

\section{MATERIALS AND METHODS}

Materials: this solar tracking system consists of the electronics and mechanical devices. Detail of those devices is: Transistor drivers $(\operatorname{Tr} 1, \operatorname{Tr} 2, \operatorname{Tr} 3$ and $\operatorname{Tr} 4$; 2N 3055), Light Emitting Diode (LED; NEC 1N1134), DC motor $(12 \mathrm{~V} / 30 \mathrm{w})$ with gear block; all resistors $(1 / 4 \mathrm{w} ; 1 \%$ error).

Methods: When the prototype of a sun tracking system is done. It shows in Fig. 11. Then, testing characteristic of the system is conducted consequently. Firstly, determine responses time or sensitivity of the system when 3 difference positions lamp is appeared, $15^{\circ}, 50^{\circ}$ and $90^{\circ}$ respectively. Then, measure the error of the sun tracking angle under variable sky condition. And finally, evaluate the total sun tracking angle error. 


\section{RESULTS}

According to Fig. 8-10, the graph illustrates the signal at the pole of the altitude motor of the solar parabolic tracking device. The oscilloscope indicates that the vertical axis is the pressure and the horizontal axis is time. From the experiment testing section, we adopt the bulbs in three directions; 15,50 and $90^{\circ}$, respectively. The result of time response and speed are as shown in Table 2.

The average of time response of the system is at approximately $37^{\circ} \mathrm{min}^{-1}$ and the speed of it position tracking is $0.3 \mathrm{sec}$.

Table 2: Experimental result

\begin{tabular}{lll}
\hline Position of lamp $\left({ }^{\circ}\right)$ & $\begin{array}{l}\text { Sensitivity to } \\
\text { the target }(\mathrm{sec})\end{array}$ & $\begin{array}{l}\text { Respond time } \\
\text { of the system }(\mathrm{sec})\end{array}$ \\
\hline 15 & 0.3 & 0.40 \\
50 & 0.3 & 1.43 \\
90 & 0.3 & 2.42 \\
\hline
\end{tabular}

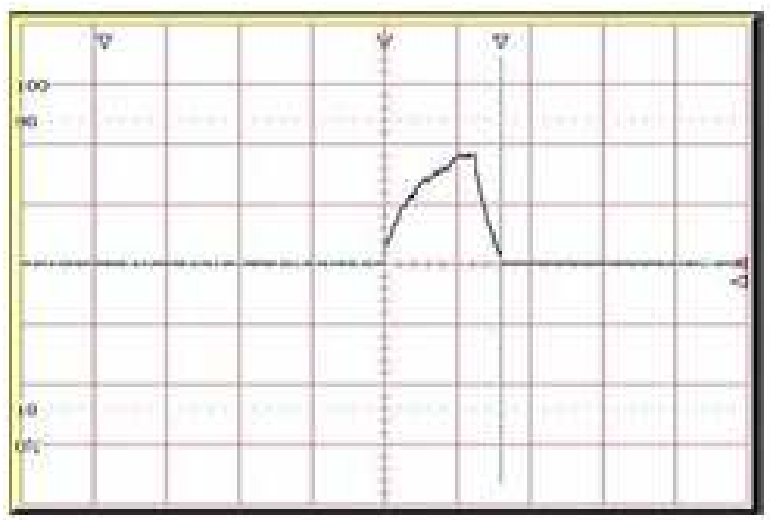

Fig. 8: Motor driving voltage for $15^{\circ}$ angle, (5V/div in vertical)

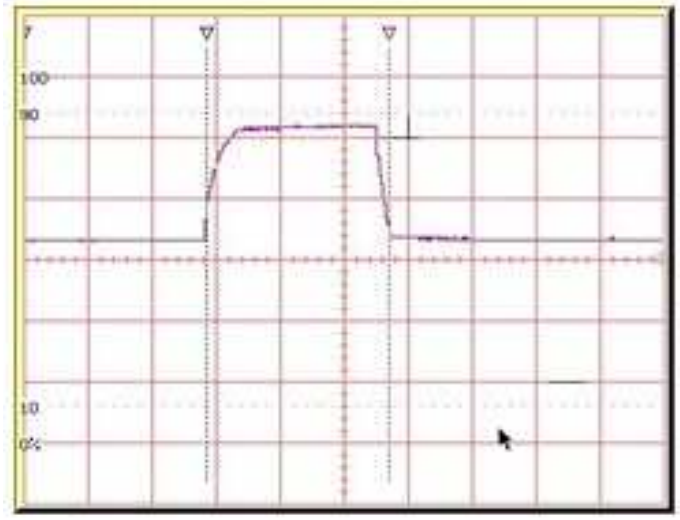

Fig. 9: Motor driving voltage for $50^{\circ}$ angle, (5 V/div in vertical)
The system evaluation by placing this device to the 'ON' status permanently, under the circumstance of clear sky condition, manifests that there is only $2.5^{\circ}$ erroneous angle in the system. This experiment testing session is conducted three days from 7 am to $6 \mathrm{pm}$ of May 2, 3 and 4, 2009. Note that on the certain dates, the condition of the sky is clear for the rest of the day. The least error that we have obtained is $0.3^{\circ}$ and the most error is at approximately $5^{\circ}$ as shown in Fig. 12.

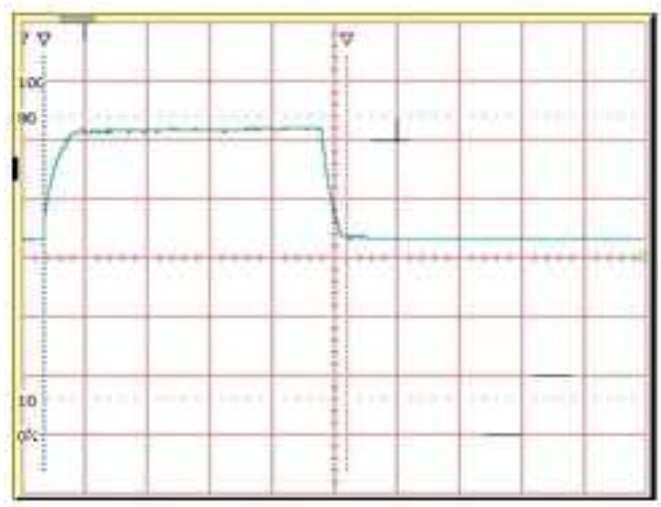

Fig. 10: Motor driving voltage for $90^{\circ}$ angle, $(5 \mathrm{~V} / \mathrm{div}$ in vertical)

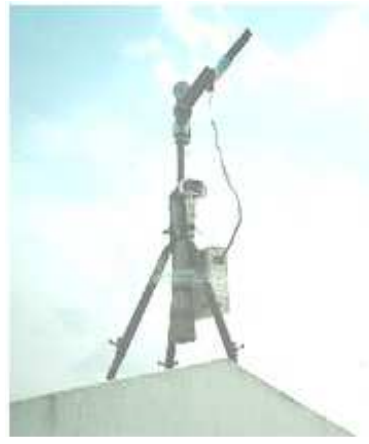

Fig. 11: Solar tracking system conducting on the roof top building

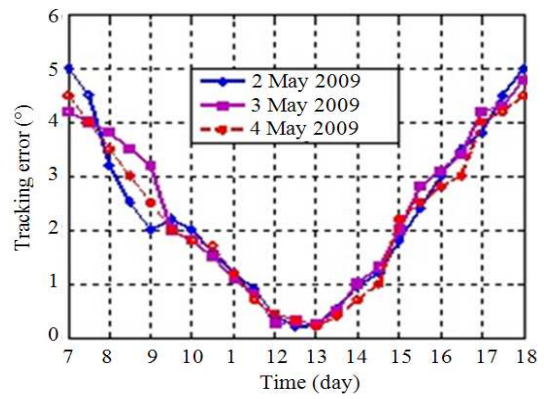

Fig. 12: Solar tracking error comparison in degree 


\section{DISCUSSION}

The sun tracking system which is proposed in this article is able to track the sun under clear sky and partly cloudy sky. In worse case, this tracking system disable when there have not a sun under the overcast sky. The maximum sun tracking error appears in daytime during low solar altitude angle (morning and evening). However, the sun tracking error decreases when the solar altitude angle increases. And the error is less than $1 \%$ when tracking angle approach the zenith. The advantage of this research is able to apply in practical work. In the future, improving a precision and an accuracy of this system would have to develop.

\section{CONCLUSION}

From the experiment, we have come to a conclusion that the system respond in terms of time at $37^{\circ} \mathrm{min}^{-1}$. This is considered as the most progress result from the past research. The accuracy of solar position tracking function satisfied our goal as well. There is only an average of $2.5^{\circ}$ error shown. Assuming the velocity rate of the sun is averagely $15^{\circ} \mathrm{h}^{-1}$, the parabolic solar tracker must track the solar energy at about every 10 min consecutively. The experiment also shows that the error rate diminishes as the solar radiance expand. However, the device might not be as perfectly exact in the angle position of the solar radiance. However, we hope to develop this device to be more exact in the position.

\section{ACKNOWLEDGMENT}

The researcher would like to express their sincere thanks to the Solar Energy and Power Electronics Research Unit, Faculty of Engineering and Research affair division of Mahasarakham University for the financial support.

\section{REFERENCES}

ElGizawy, M., A. Noureldin, J. Georgy, U. Iqbal and N. El-Sheimy, 2010. Wellbore surveying while drilling based on Kalman filtering. Am. J. Eng. Applied Sci., 3: 240-259. http://www.scipub.org/fulltext/ajeas/ajeas32240259.pdf

Kalogirou, S.A., 1996. Design and construction of a one- axis sun-tracking. Solar Energy, 57: 465-469. DOI: 10.1016/S0038-092X(96)00135-1

Konar, A. and A.K. Mandal, 1991. Microprocessor base automatic sun tracker. IEE Proc. A. Measure. Technol. $\quad$ Sci., 138: 237-241. http://ieeexplore.ieee.org/xpl/freeabs_all.jsp?arnum ber $=254047$

Koyuncu, B. and K. Balasubramanian, 1991. A microprocessor controlled automatic sun tracker. IEEE Trans. Consumer Elect., 37: 913-917. DOI: $10.1109 / 30.106958$

Marghany, M. and M. Hashim, 2009. Robust of Doppler centroid for mapping sea surface current by using radar satellite data. Am. J. Eng. Applied Sci., 2: 781-788. http://www.scipub.org/fulltext/ajeas/ajeas24781788.pdf

Roth, P., A. Georgiev and H. Boudinov, 2004. Design and construction of a system for sun-tracking. Renew. Energy, 29: 393-402. DOI: 10.1016/S09601481(03)00196-4 